\title{
On Revenue in the Generalized Second Price Auction
}

\author{
Brendan Lucier \\ Dept of Computer Science \\ University of Toronto \\ blucier@cs.toronto.edu
}

\author{
Renato Paes Leme \\ Dept of Computer Science \\ Cornell University \\ renatoppl@cs.cornell.edu
}

\author{
Éva Tardos ${ }^{\dagger}$ \\ Dept of Computer Science \\ Cornell University \\ eva@cs.cornell.edu
}

\begin{abstract}
The Generalized Second Price (GSP) auction is the primary auction used for selling sponsored search advertisements. In this paper we consider the revenue of this auction at equilibrium. Most previous work on the revenue of GSP focuses on envy free equilibria of the full information version of this game. Envy-free equilibria are known to obtain at least the revenue of the VCG auction. Here we consider revenue in equilibria that are not envy-free, as well as in equilibria for the Bayesian, partial information version of this game.
\end{abstract}

We show that, at any Nash equilibrium of the full-information game, the GSP auction obtains at least half of the revenue of the VCG mechanism excluding the payment of a single participant. This bound is tight, and we give examples demonstrating that GSP cannot approximate the full revenue of the VCG mechanism either in the full information game, or in the Bayesian partial information game (even if agent values are independently drawn from identical uniform distributions).

We then consider the revenue properties of the GSP auction with reserve prices. We prove that if agent values are drawn from a distribution satisfying the monotone hazard rate assumption, then the GSP auction paired with an appropriate reserve price generates a constant fraction (approximately 6.06) of the optimal revenue. We also show that the GSP revenue without reserve prices approximates the VCG revenue in the Bayesian game when the click-through rates are well separated.

Finally, we consider the tradeoff of maximizing revenue and social welfare. We introduce a natural convexity assumption on the click-through rates, and show that it implies that the revenue-maximizing equilibrium of GSP in the full information model will necessarily be envy-free. In particular, it is possible to maximize revenue and social welfare simultaneously. On the other hand, without this convexity assumption, we demonstrate that revenue may be maximized at a non-envy-free equilibrium that generates a socially inefficient allocation.

\section{Categories and Subject Descriptors}

\footnotetext{
*Supported in part by NSF grants CCF-0729006.

†Supported in part by NSF grants CCF-0910940 and CCF0729006, ONR grant N00014-98-1-0589, a Yahoo! Research Alliance Grant, and a Google Research Grant.
}

J.4 [Social and Behavioral Sciences]: Economics; F.2.2 [Analysis of Algorithms and Problem Complexity]: Nonnumerical Algorithms and Problems

\section{General Terms}

games, economics, algorithms

\section{Keywords}

sponsored search auctions, revenue

\section{INTRODUCTION}

The sale of sponsored search advertising space is a primary source of revenue for Internet companies, and responsible for billions of dollars in annual advertising revenue [7]. The Generalized Second Price (GSP) auction is the premier method by which sponsored search advertising space is sold; it is currently employed by Google, Bing, and Yahoo. However, use of the GSP auction is not universal: the classical VCG mechanism was recently adopted by Facebook for its AdAuction system [12]. In fact, Google also considered switching its advertising platform to a VCG auction some years ago, but eventually decided against it [19]. This apparent tension begs the question of how these mechanisms compare. There are many factors in comparing possible mechanisms: The welfare of three distinct user groups (the experience of the searchers, the welfare of advertisers, and the revenue of the auction) are all important considerations, as well as the simplicity of the auction design. In this paper, we take the point of view of the seller and compare the revenue properties of the GSP and VCG auctions.

Let us first briefly describe a simple model of the market, introduced by Edelman et al [7] and Varian [20]. In sponsored search, a user makes a query for certain keywords in a search engine and is presented with a list of relevant advertisements in addition to organic search results. We assume a "pay-perclick" pricing model, in which the advertiser pays a fee to the search provider whenever a user clicks on an advertisement. There are multiple positions (or "slots") in which an ad may appear, and the probability that a user clicks an ad depends on its slot. This is modeled as a click-through rate (CTR) associated with each slot, that being the probability of getting a click for an advertisement in that position. The search engine must therefore determine which ads to place where, and determine a price per click for each slot. This is done via an auction in which advertisers make bids, which are viewed as the advertiser's maximum willingness to pay per click. We note that this simplified model as a one-shot 
game does not account for advertiser budgets, so models the case when budgets are large. Also, for simplicity of presentation, we will assume that all ads have the same quality (i.e. click-through rate does not depend on the advertiser selected for a slot), though our results extend to the version of the model with separable click-though rates.

The VCG and GSP mechanisms differ in the way in which the aforementioned auction is resolved. In both auctions, advertisers are assigned slots in order of their bids, with higher bidders receiving slots with higher CTRs. The two auctions differ in their payment schemes. In VCG, each agent pays an amount equal to his externality on the other agents: the decrease in the total welfare of all other agents caused by the presence of this advertiser. By contrast, in GSP each advertiser simply pays a price per click equal to the next highest bid. The VCG auction has the strong property of being truthful in dominant strategies. The GSP auction is not truthful, and is therefore prone to strategic bidding behavior. Indeed, strategic manipulation of bids is welldocumented in historical GSP bidding data [6].

Since the VCG mechanism is truthful, the revenue of VCG is simply the revenue generated when all bidders declare their values truthfully. If bidders declare their values truthfully in a GSP auction, GSP generates strictly more revenue than VCG. However, rational agents may not declare their values truthfully when participating in a GSP auction. Thus, when studying the revenue of GSP, we consider the revenue generated at a Nash equilibrium; that is, a profile of bidding strategies such that no advertiser can improve his utility by unilaterally deviating. Our goal, then, is a comparison between the revenue of the VCG auction and the revenue of GSP at equilibrium. Note that since there will not be a unique Nash equilibrium of GSP in general, there may be many possible revenue amounts generated by GSP.

This basic model of the GSP auction was introduced by Edelman et al [7] and Varian [20]. Both papers consider the full information case, and study a more restrictive notion of equilibrium than Nash, which they call envy-free or symmetric equilibrium respectively. They show that all envy-free equilibria are efficient and generate at least as much revenue as the VCG auction. Both [7] and [20] present informal arguments to support the equilibrium selection for this class of equilibria, but there is no strong theoretical model that explains this selection $[4,9]$. Further, the notion of envy-free equilibria applies only in the full information game. In fact [11] shows that an efficient equilibrium may not exist in the Bayesian game (even when the valuations are drawn from identical uniform distributions). However, for the broader class of all Nash equilibria, the revenue properties of GSP are not understood. The primary focus of this paper is to study the revenue of the GSP auction, in relation to VCG, over the set of all Nash equilibria (including inefficient equilibria). We ask: how is the revenue of GSP affected if one cannot assume that agents necessarily converge to an envyfree equilibrium?

In Sections 3 and 5 we consider the full information game. For many keywords the search auction is repeated many times each day. In general, the repeated nature of this auction allows for complex strategic interactions. However, if agents learn each others' bidding preferences over time, and converge to a stable bidding pattern, then this outcome is well modeled by a full-information Nash equilibrium of the one-shot game.

In Section 4 we consider the Bayesian version of this game. For many keywords, the ability of a player to exactly predict his opponents types is impaired. Due to complicating factors such as the budgets, quality scores (which depend on many characteristics of each query, such as origin, time, search history of the user), and the underlying ad allocation algorithm, each auction is different (even those triggered by the same search term). To capture this measure of uncertainty, one might consider equilibria in a Bayesian partial information model, to determine whether these sources of uncertainty affect auction revenue.

A common tool for increasing revenue in settings of partial information is to apply reserve prices, where bids are rejected unless they meet some minimum bid $r$. Indeed, when bidders' values are identically distributed from a distribution satisfying the regularity condition ${ }^{1}$, the revenue-optimal truthful auction for our sponsored search market is the VCG auction with an appropriate reserve price [17]. Edelman and Schwarz [8] show that in GSP auctions reserve prices have a surprisingly large effect on revenue.

Results. We begin by considering lower bounds on the revenue generated by GSP. One might wish to bound the revenue of GSP with respect to the revenue of VCG, but we demonstrate that the revenue of GSP at equilibrium may be arbitrarily less than that of VCG. However, we can bound the revenue of GSP with respect to a related benchmark: we prove that at any Nash equilibrium, the revenue generated by GSP is at least half of the VCG revenue, excluding the single largest payment of a bidder. Thus, as long as the VCG revenue is not concentrated on the payment of a single participant, the worst-case GSP revenue approximates the VCG revenue to within a constant factor. Furthermore, this result also holds when an arbitrary reserve price is set upon the sale of a slot. We also provide an example illustrating that the factor of 2 in our analysis is tight.

One might hope that the gap between GSP and VCG revenue is an artifact of agents having very different values, or an artifact of the full-information setting. To the contrary, we demonstrate that this gap is prevalent in a broad setting: even in a partial information setting where agents' values are drawn independently from identical uniform distributions, the gap between the VCG revenue and GSP revenue at Bayesian-Nash equilibrium can be arbitrarily large.

However, we show that if we allow the auctioneer to include reserve prices the GSP auction always obtains a constant fraction of the VCG revenue. Recall that if agent values are drawn identically from a distribution that satisfied the monotone hazard rate assumption, then it is well-known, that the revenue-optimal truthful mechanism is the VCG auction with Myerson's reserve price [17]. We prove that,

\footnotetext{
${ }^{1}$ Many common distributions are regular, including all uniform, normal, and exponential distributions.
} 
assuming monotone hazard rates, the GSP auction paired with this same Myerson reserve price obtains a constant fraction of the optimal revenue, where our constant is $c \approx 6.06$. We emphasize that this is the first such bound on the revenue obtained by GSP with reserve prices. Our analysis makes use of known bounds for the social welfare generated by GSP at Bayes-Nash equilibrium [5, 16], so any improvement in those bounds would translate into a corresponding improvement in our revenue bound.

Further, we also show that without using reserve prices, if the slot CTRs satisfy a certain well-separatedness condition - namely that the CTRs of adjacent slots differ by at least a certain constant factor - then we prove that GSP does obtain a constant fraction of the VCG revenue even in settings of partial information, extending a result of Lahaie [13] who considered welfare under this assumption on the CTRs. This result holds without assuming that agents avoid dominated strategies, as long as there are at least three participants in the auction.

We then return to the full-information game and analyze the tradeoffs of the maximum revenue attainable by the GSP mechanism under different equilibrium notions. We demonstrate that there can exist inefficient, non-envy-free equilibria that obtain greater revenue than any envy-free equilibrium. However, we prove that if CTRs are convex, meaning that the marginal increase in CTR is monotone in slot position, then the optimal revenue always occurs at an envy-free equilibrium. This implies that when click-through rates are convex, the GSP auction optimizes revenue at an equilibrium that simultaneously maximizes the social welfare. We feel that the convexity assumption is quite natural; note that this assumption is satisfied in the case when CTRs degrade by a constant factor from one slot to the next.

Related Work. There has been considerable amount of work on the economic and algorithmic issues behind sponsored search auctions - see an early survey of Lahaie et al [14] for an overview. The GSP model we adopt is due to Edelman et al [7], Varian [20] and Aggarwal et al [2]. Edelman et al and Varian consider a more restrictive notion of equilibrium than Nash. Edelman et al calls it envy-free equilibrium and Varian calls it symmetric equilibrium. Both authors show that this class of equilibria produce always optimal social welfare and revenue at least as good as the revenue of VCG.

Varian [21] shows how to compute the revenue optimal envy free Nash equilibrium, however in his model, he allows agents to overbid (which is dominated strategy, and we consider it unnatural). We consider the question of maximum revenue equilibria without the assumption of envy-free outcome. We show that in general inefficient equilibria can generate more revenue than efficient ones. However, under a natural convexity assumption on click-through rates, we show that the maximum revenue equilibrium is envy free, and hence efficient, and show how to compute it efficiently.

Edelman and Schwarz [9] model the repeated auctions for a keyword as a repeated game, and show using Myerson's optimal auction [17] that if valuations are drawn from an iid distribution, than the Nash equilibria that arise as a sta- ble limit of rational play in this repeated game, cannot have revenue more than the optimal auction: VCG with an appropriately chosen reserve price. This leaves open the question whether GSP may generate revenue much less than the VCG auction, which is the main question we consider. However, unlike Edelman and Schwarz [9] we do not model a repeated game explicitly, as rational play in a repeated game is very complex. Rather, we consider all stable outcomes of the auction, not only those that arise as limits of rational repeated play, which makes our results more general.

Gomes and Sweeney [11] study GSP as a Bayesian game - analyzing the symmetric efficient equilibria of this auction. They analyze the influence of click-through-rates in the revenue and observe the counter-intuitive phenomenon by which revenue decreases when click-through-rates increase. They also discuss the influence of reserve prices.

Paes Leme and Tardos [18] showed that the social welfare of GSP in equilibrium is within a constant factor of the optimal social welfare - which is composed by the engine revenue and the players total surplus. Lucier and Paes Leme [16] and Caragiannis et al [5] recently improved the bounds. In the present work we tackle a natural question arising from their work: even though GSP guarantees reasonably high welfare, how does this welfare guarantee break down into its revenue and total surplus components?

There has been considerable work focused on studying revenue properties of GSP either by analyzing real auction data or by running simulations. Athey and Nekipelov [3] study the effect of quality-factors uncertainty in the revenue. Lahaie [13], Lahaie and Pennock [15] and Feng et al [10] study the effect of different ranking functions. Borgers et al [4] study revenue for alternative auction formats. Edelman and Schwarz [8] study the effect of reserve prices.

Our results compare the revenue of different mechanisms at equilibrium. It is worth noting that the well-known revenue equivalence theorem, which provides conditions under which alternative mechanisms generate the same revenue at equilibrium, does not apply in our settings. Revenue equivalence requires that agents have values drawn from identical distributions and the mechanisms generate the same outcome. As a result, this equivalence does not apply in the full information setting, and cannot be used to compare inefficient equilibria of GSP to the VCG revenue.

\section{PRELIMINARIES}

An AdAuctions instance is composed of $n$ players and $n$ slots. In the full information model, ach player has a value $v_{i}$ for each click he gets, and each slot $j$ has click-throughrate $\alpha_{j}$. That means that if player $i$ is allocated in slot $j$, he gets $\alpha_{j}$ clicks in expectation. We assume we number players such that $v_{1} \geq v_{2} \geq \ldots \geq v_{n}$ and $\alpha_{1} \geq \ldots \geq \alpha_{n}$. Let $\alpha=\left(\alpha_{1}, \ldots, \alpha_{n}\right)$ be the CTR vector and $\mathbf{v}=\left(v_{1}, \ldots, v_{n}\right)$ be the type vector. Our results also extend to the model when players also have a known quality factor $\gamma_{i}$, and if player $i$ is allocated in slot $j$, he gets $\gamma_{i} \alpha_{j}$ clicks in expectation, however, for the rest of the paper, we assume that $\gamma_{i}=1$ for clarity of exposition.

A mechanism for the AdAuctions problem has the following 
form: Since valuations $v_{i}$ are private information, it begins by eliciting some bid $b_{i}$ for the players, which works as his "declared valuation". We write $\mathbf{b}=\left(b_{1}, \ldots, b_{n}\right)$ for the bid vector. Using the $\mathbf{b}$ and $\alpha$, the mechanism chooses an allocation $\pi:[n] \rightarrow[n]$ which means that player $\pi(j)$ is allocated to slot $j$, and a price vector $\mathbf{p}=\left(p_{1}, \ldots, p_{n}\right)$, where $p_{i}$ is the price that player $i$ pays for click. Player $i$ then, experiences utility $u_{i}(\mathbf{b})=\alpha_{\sigma(i)}\left(v_{i}-p_{i}\right)$, where $\sigma(i)=\pi^{-i}(i)$.

The social welfare generated by the mechanism is given by $S W(\mathbf{v}, \pi)=\sum_{i} \alpha_{i} v_{\pi(i)}$ and the revenue is given by $\mathcal{R}(\mathbf{b})=$ $\sum_{i} \alpha_{\sigma(i)} p_{i}$. We focus on two mechanisms: GSP and VCG: in both mechanisms, the players are ordered by their bids, i.e, $\pi(j)$ is the player with the $j^{\text {th }}$ largest bid, but they differ in the payments charged. GSP mimics the single-item second price auction by charging each player the bid of the next highest bidder, i.e. $p_{i}=b_{\pi(\sigma(i)+1)}$ if $\sigma(i)<n$ and zero otherwise. VCG charges each player the externality it imposes on the other players, which is

$$
p_{i}^{V C G}=\frac{1}{\alpha_{\sigma(i)}} \sum_{j=\sigma(i)+1}^{n}\left(\alpha_{j-1}-\alpha_{j}\right) b_{\pi(j)} .
$$

If the bidders truthfully declare their valuation in both VCG and GSP, then GSP generates strictly more revenue, as the revenue associated with player $i$ in VCG is

$$
p_{i}^{V C G} \alpha_{\sigma(i)}=\sum_{j=\sigma(i)+1}^{n}\left(\alpha_{j-1}-\alpha_{j}\right) b_{\pi(j)} \leq \alpha_{\sigma(i)} b_{\sigma(i)+1},
$$

and this upper bound is the GSP price paid by player $i$. VCG has the remarkable property that regardless of what the other players are doing, it is a weakly dominant strategy for player $i$ to report his true valuation. The resulting outcome of VCG is therefore social-welfare optimal and the revenue is

$\mathcal{R}^{V C G}(\mathbf{v})=\sum_{i} \sum_{j>i}\left(\alpha_{j-1}-\alpha_{j}\right) v_{j}=\sum_{i=2}^{n}(i-1)\left(\alpha_{i-1}-\alpha_{i}\right) v_{i}$.

The GSP auction, however, is not truthful. Thus, for GSP, we are interested in the set of bid profiles that constitute a Nash equilibrium, i.e. such that

$$
u_{i}\left(b_{i}, \mathbf{b}_{-i}\right) \geq u_{i}\left(b_{i}^{\prime}, \mathbf{b}_{-i}\right), \forall b_{i}^{\prime} \in\left[0, v_{i}\right] .
$$

We will assume that players do not overbid (i.e. that $b_{i} \leq$ $v_{i}$ ) since bidding more than one's true value is a weakly dominated strategy [18].

We say that an equilibrium is efficient if it maximizes social welfare, which occurs when $\pi(i)=i$ for all $i$.

We will also consider this comparison in the presence of a reserve price. Let $\mathrm{VCG}_{r}$ be the VCG mechanism with reserve price $r$, where we discard all players with bids smaller then $r$ and run the VCG mechanism on the remaining players, who then pay price per click $\max \left\{p_{i}, r\right\}$. In the analogous variant of the GSP mechanism, which we call GSP with reserve price $r\left(\mathrm{GSP}_{r}\right)$, we also discard all players with bids smaller then $r$, the remaining players are allocated using GSP, and the last player to be allocated pays price $r$ per click.

\subsection{Equilibrium hierarchy for GSP}

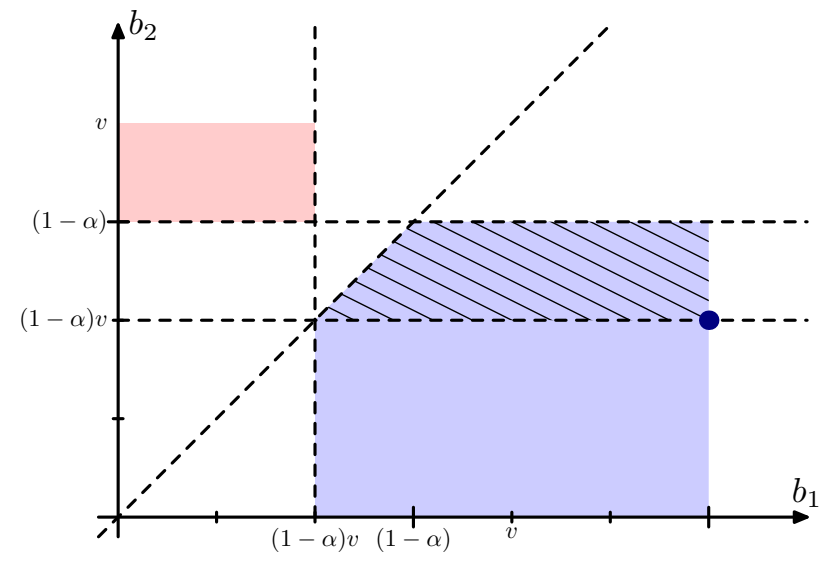

Figure 1: Equilibria hierarchy for GSP for $\alpha=$ $[1,1 / 2], v=[1,2 / 3]$ : the strong blue dot represents the VCG outcome, the pattern region the envy-free equilibria, the blue region all the efficient equilibria and the red region the inefficient equilibria

Edelman, Ostrovsky and Schwarz [7] and Varian [20] showed that the full information game always has a Pure Nash equilibrium, and moreover, there is a pure Nash equilibrium which has same outcome and payments as VCG. At this equilibrium, players bid

$$
b_{i}^{V}=\frac{1}{\alpha_{i-1}} \sum_{j=i}^{n}\left(\alpha_{j-1}-\alpha_{j}\right) v_{j} .
$$

The authors also define a class of equilibria called envy-free or symmetric equilibria. This is the class of bid profiles b such that

$$
\alpha_{\sigma(i)}\left(v_{i}-b_{\sigma(i)+1}\right) \geq \alpha_{j}\left(v_{i}-b_{j+1}\right) .
$$

It is easy to see that all envy-free equilibria are Nash equilibria, though not all Nash equilibria are envy-free. The bid profiles that are envy-free are always efficient and the revenue of an envy-free equilibrium is always greater than or equal to the VCG revenue. That is, if $\mathbf{b}$ is an envy-free equilibrium, $\mathcal{R}(\mathbf{b}) \geq \mathcal{R}^{V C G}(\mathbf{v})$.

Although all envy-free equilibria are efficient, there are efficient equilibria that are not envy-free, as one can see for example in Figure 1, as well as inefficient equilibria. We therefore have the following hierarchy:

$\left\{\begin{array}{c}\text { VCG } \\ \text { outcome }\end{array}\right\} \subseteq\left\{\begin{array}{l}\text { envy-free } \\ \text { equilibria }\end{array}\right\} \subseteq\left\{\begin{array}{l}\text { efficient } \\ \text { Nash eq }\end{array}\right\} \subseteq\left\{\begin{array}{c}\text { all } \\ \text { Nash }\end{array}\right\}$

\subsection{Bayesian setting}

In a Bayesian setting, each player knows her own valuation but only knows a distribution on the other players' valuations. In this model the values of the players are random variables, with type vector $\mathbf{v}$ drawn from a known distribution $F$. After learning his own value $v_{i}$, a player chooses a bid $b_{i}\left(v_{i}\right)$ to play in the AdAuctions game. The strategies are therefore bidding functions $b_{i}: \mathbb{R}_{+} \rightarrow \mathbb{R}_{+}$, and we will continue to assume that players do not overbid, i.e. $b_{i}(v) \leq v$. A set of bidding functions is a Bayes-Nash equilibrium if, for all $i, v_{i}$, and $b_{i}^{\prime}$,

$$
\mathbb{E}\left[u_{i}\left(b_{i}\left(v_{i}\right), \mathbf{b}_{-i}\left(\mathbf{v}_{-i}\right)\right) \mid v_{i}\right] \geq \mathbb{E}\left[u_{i}\left(b_{i}^{\prime}\left(v_{i}\right), \mathbf{b}_{-i}\left(\mathbf{v}_{-i}\right)\right) \mid v_{i}\right] .
$$


A useful tool for studying revenue in the Bayesian setting is Myerson's Lemma, which can be rephrased in the AdAuctions setting as follows. Given a distribution $F$ over agent values, the virtual valuation function is defined by $\phi(x)=$ $x-\frac{1-F(x)}{f(x)}$.

Lemma 1 (Myerson [17]) At any Bayes-Nash equilibrium of an AdAuction mechanism, we have that, for all $i$,

$$
\mathbb{E}\left[\alpha_{\sigma(i)} p_{i}\right]=\mathbb{E}\left[\alpha_{\sigma(i)} \phi\left(v_{i}\right)\right]
$$

where $p_{i}$ is the payment per click of player $i$ and $\alpha_{\sigma(i)}$ is the number of clicks received by agent $i$, and expectation is with respect to $\mathbf{v} \sim \mathbf{F}$.

We say that a distribution is regular if $\phi(x)$ is a monotone non-decreasing function. For regular distributions, the revenue-optimal mechanism for AdAuctions corresponds to running VCG with Myerson's reserve price $r$, which is the largest value such that $\phi(r)=0$. We will refer to this as Myerson's mechanism, $V C G_{r}$.

Running GSP (or VCG) with reserve price $r$ means not allocating any user with value $v_{i}<r$ and running GSP (or VCG) with the remaining agents. For the allocated agents, the mechanism charges per click the maximum between the GSP (VCG) price and $r$

A special class of regular distributions is the monotone hazard rate distributions (MHR), which are the distributions for which $f(x) /(1-F(x))$ is non-decreasing. We note that all MHR distributions are regular.

\section{REVENUE IN FULL INFORMATION GSP}

The goal of this section is to compare the revenue properties of GSP and VCG. Unfortunately, there are no universal constants $c_{1}, c_{2}>0$ such that for every AdAuctions instance $\alpha, \mathbf{v}$ and for all equilibria $b$ of GSP it holds that

$$
c_{1} \cdot \mathcal{R}^{V C G}(\mathbf{v}) \leq \mathcal{R}(\mathbf{b}) \leq c_{2} \cdot \mathcal{R}^{V C G}(\mathbf{v}) .
$$

In fact, GSP can generate arbitrarily more revenue than VCG and vice-versa. For example, consider two players with $\alpha=\{1,0\}, \mathbf{v}=\{2,1\}$. Then VCG generates revenue 1 , but GSP has the Nash equilibrium $\mathbf{b}=[2,0]$ that generates no revenue.

As a counter-example for the second inequality, consider the following instance: $\alpha=\{1,1-\epsilon\}, \mathbf{v}=\left\{\epsilon^{-1}, 1\right\}$. Notice that the revenue produced by VCG is $\epsilon$, while GSP has the equilibrium $\mathbf{b}=[1,1]$ generating revenue 1 .

We will prove that the GSP revenue cannot be much less than a revenue benchmark based upon the VCG auction. Intuitively, the difficulty behind our bad examples above is in extracting revenue from the player with the largest private value. Motivated by this, we consider the following benchmark:

$$
\begin{aligned}
\mathcal{B}(\mathbf{v}) & =\sum_{i=2}^{n} p_{i}^{V C G} \alpha_{\sigma(i)} \\
& =\sum_{i=2}^{n} \sum_{j>i}\left(\alpha_{j-1}-\alpha_{j}\right) v_{j}=\sum_{i=2}^{n}(i-2)\left(\alpha_{i-1}-\alpha_{i}\right) v_{i}
\end{aligned}
$$

which is the VCG revenue from players $2,3, \ldots, n$. We show that the GSP revenue is always at least half of this benchmark at any equilibrium. Thus, unless VCG gets most of its revenue from the first player, GSP revenue will be within a constant factor of the VCG revenue.

Theorem 2 Given an AdAuctions instance $\alpha, \mathbf{v}$, and a Nash equilibrium $\mathbf{b}$ of $G S P$, we have $\mathcal{R}(\mathbf{b}) \geq \frac{1}{2} \mathcal{B}(\mathbf{v})$, and this bound is tight.

We prove Theorem 2 in two steps. First we define the concept of up-Nash equilibrium for GSP, then we show that any inefficient Nash equilibria can be written as an efficient upNash equilibrium. In the second step, we prove the desired revenue bound for all efficient up-Nash equilibria.

Definition 3 Given a bid profile $\mathbf{b}$, we say it is $\mathbf{u p}$-Nash for player $i$ if he can't increase his utility by taking some slot above, i.e.

$$
\alpha_{\sigma(i)}\left(v_{i}-b_{\pi(\sigma(i)+1)}\right) \geq \alpha_{j}\left(v_{i}-b_{\pi(j)}\right), \forall j<\sigma(i) .
$$

Analogously, we say that $\mathbf{b}$ is down-Nash for player $i$ if he can't increase his utility by taking some slot below, i.e.

$$
\alpha_{\sigma(i)}\left(v_{i}-b_{\pi(\sigma(i)+1)}\right) \geq \alpha_{j}\left(v_{i}-b_{\pi(j+1)}\right), \forall j>\sigma(i) .
$$

A bid profile is up-Nash (down-Nash) if it is up-Nash (downNash) for all players i. Clearly a bid profile b is a Nash equilibrium iff it is both up-Nash and down-Nash.

Lemma 4 If a bid profile $\mathbf{b}$ is a Nash equilibrium, then the bid profile $\mathbf{b}^{\prime}$ where $b_{i}^{\prime}=b_{\pi(i)}$ is up-Nash.

Proof. We will prove the lemma by modifying bid profile b in a sequence of steps. Fix some $k \leq n$, and suppose that $\mathbf{b}$ is a bid profile (with corresponding allocation $\pi$ ) such that

- players $j=1, \ldots, k$ satisfy the Nash conditions (i.e. both up-Nash and down-Nash) in $\mathbf{b}$,

- players $j=k+1, \ldots, n$ are such that $\sigma(j)=j$ and they satisfy the up-Nash conditions in $\mathbf{b}$, and

- $\sigma(k)<k$.

We then define $\mathbf{b}^{\prime}$ by swapping the bids of players $k$ and $\pi(k)$, that is setting $b_{i}^{\prime}=b_{i}$ for $i \neq k, \pi(k), b_{k}^{\prime}=b_{\pi(k)}$, and $b_{\pi(k)}^{\prime}=b_{k}$. We claim that $\mathbf{b}^{\prime}$ is up-Nash for players $k, \ldots, n$ and Nash for the remaining players. This then implies the desired result, since we can apply this operation for $k=n$, followed by $k=n-1, \ldots, 2$, resulting in the required bid profile.

Since our transformation does not alter the bids associated with given slots, we just need to check three things: the up and down-Nash inequalities for player $\pi(k)$, and the up-Nash inequality for player $k$.

Under bid profile $\mathbf{b}^{\prime}$, player $\pi(k)$ gets slot $\sigma(k)$. This player doesn't want to change his bid to win any slot $j>\sigma(k)$ since 
in the bid profile b player $k$ with lower value didn't want to get these slots. We therefore have

$$
\alpha_{\sigma(k)}\left(v_{k}-b_{\pi(\sigma(k)+1)}\right) \geq \alpha_{j}\left(v_{k}-b_{\pi(j+1)}\right)
$$

and since $v_{\pi(k)} \geq v_{k}$, we conclude

$$
\alpha_{\sigma(k)}\left(v_{\pi(k)}-b_{\pi(\sigma(k)+1)}\right) \geq \alpha_{j}\left(v_{\pi(k)}-b_{\pi(j+1)}\right) .
$$

To see that player $\pi(k)$ would not prefer to take any slot $j<\sigma(k)$, notice that $\pi(k)$ didn't want to move to a higher slot in $b$, so

$$
\alpha_{k}\left(v_{\pi(k)}-b_{\pi(k+1)}\right) \geq \alpha_{j}\left(v_{\pi(k)}-b_{\pi(j)}\right) .
$$

This, combined with equation (1) for $j=k$ (stating that $\pi(k)$ prefers slot $\sigma(k)$ to $k$ ) gives us the up-Nash inequality for player $\pi(k)$.

Next consider player $k$ in bid profile $\mathbf{b}^{\prime}$, where we gets slot $k$. We wish to prove the up-Nash inequality for $k$. Notice that, in $\mathbf{b}, \pi(k)$ had slot $k$ and didn't want to switch to a higher slot, so we know

$$
\alpha_{k}\left(v_{\pi(k)}-b_{\pi(k+1)}\right) \geq \alpha_{j}\left(v_{\pi(k)}-b_{\pi(j)}\right) .
$$

Now, since $v_{\pi(k)} \geq v_{k}$, we have

$$
\alpha_{k}\left(v_{k}-b_{\pi(k+1)}\right) \geq \alpha_{j}\left(v_{k}-b_{\pi(j)}\right)
$$

which is the desired inequality.

Proof of Theorem 2 : Given any Nash equilibrium b, consider the bid profile $\mathbf{b}^{\prime}$ of Lemma 4 , which is an up-Nash equilibrium in which each player $k$ occupies slot $k$. By the up-Nash inequalities, for each $k$ we have

$$
\alpha_{k}\left(v_{k}-b_{k+1}^{\prime}\right) \geq \alpha_{k-1}\left(v_{k}-b_{k-1}^{\prime}\right) .
$$

We can rewrite this as

$$
\alpha_{k-1} b_{k-1}^{\prime} \geq\left(\alpha_{k-1}-\alpha_{k}\right) v_{k}+\alpha_{k} b_{k+1}^{\prime} .
$$

Then, since $\alpha_{k} \geq \alpha_{k+1}$,

$$
\alpha_{k-1} b_{k-1}^{\prime} \geq \sum_{j \in k+2 \mathbb{N}}\left(\alpha_{j-1}-\alpha_{j}\right) v_{j}
$$

where $k+2 \mathbb{N}=\{k, k+2, k+4, \ldots\}$. Now we can bound the revenue of $\mathbf{b}$ :

$$
\begin{aligned}
\mathcal{R}(\mathbf{b})=\mathcal{R}\left(\mathbf{b}^{\prime}\right) & =\sum_{k} \alpha_{k} b_{k+1}^{\prime} \geq \sum_{k} \alpha_{k+1} b_{k+1}^{\prime} \geq \\
& \geq \sum_{k} \sum_{j \in k+2+2 \mathbb{N}}\left(\alpha_{j-1}-\alpha_{j}\right) v_{j} \geq \\
& \geq \sum_{k=2}^{n} \frac{k-2}{2}\left(\alpha_{k-1}-\alpha_{k}\right) v_{k}=\frac{1}{2} \mathcal{B}(\mathbf{v}) .
\end{aligned}
$$

To show that the bound in Theorem 2 is tight, consider the following example with $n$ slots and $n$ players, parametrized by $\delta>0$ :

$$
\begin{gathered}
\alpha=[1,1,1,1, \ldots, 1,1-\delta, 0], \\
\mathbf{v}=[1,1,1,1, \ldots, 1,1, \delta], \\
\mathbf{b}=[\delta, \delta, \delta, \delta, \ldots, \delta, \delta, 0] .
\end{gathered}
$$

In this case $\mathcal{R}(\mathbf{b})=(n-2) \delta+\delta(1-\delta)$ and $\mathcal{R}^{V C G}(\mathbf{v})=$ $\left(2 \delta-\delta^{2}\right)(n-3)+\delta(1-\delta)$. Therefore $\lim _{n \rightarrow \infty} \frac{\mathcal{R}(\mathbf{b})}{\mathcal{B}(\mathbf{v})}=2-\delta$ and it tends to 2 as $\delta \rightarrow 0$.

Notice that those bounds also carry for the case where there is a reserve price $r$. We compare the revenue $\mathcal{R}_{r}(\mathbf{b})$ with reserve price $r$, against a slightly modified benchmark: $\mathcal{B}_{r}(\mathbf{v})$ which is the revenue $\mathrm{VCG}_{r}$ extracts from players $2, \ldots, n$.

Corollary $\mathbf{5}$ Let $\mathbf{b}$ be a Nash equilibrium of the GSP $P_{r}$ game, then $\mathcal{R}_{r}(\mathbf{b}) \geq \frac{1}{2} \mathcal{B}_{r}(\mathbf{v})$.

Proof. We can assume wlog that $v_{i}, b_{i} \geq r$ (otherwise those players don't participate in any of the auctions). We can define an upper-Nash bid profile $\mathbf{b}^{\prime}$ as in Lemma 4. Now, notice that all players in $\mathbf{b}^{\prime}$ are paying at least $r$ per click. We can divide the players in two groups: players $1 \ldots k$ are paying more than $r$ in $\mathrm{VCG}_{r}$ and players $k+1 \ldots n$ are paying exactly $r$. It is trivial that for the players $k+1 \ldots n$ we extract at least the same revenue under $\mathrm{VCG}_{r}$ then under $\mathrm{GSP}_{r}$. For the rest of the players we need to do the exact same analysis as in the proof of Theorem 2 .

\section{REVENUE IN THE BAYESIAN SETTING}

We showed in the full information setting that there are AdAuctions instances $\alpha, \mathbf{v}$ such that VCG generates positive revenue and there are GSP equilibria generating no revenue. We show in this section that this can be seen as an artifact of the full-information setting: if one assumes that valuations come iid from a well-behaved distribution and we set the proper reserve price in GSP, then we are guaranteed to extract a constant fraction of the revenue.

The following example shows that setting the optimal reserve price is indeed necessary:

Example. We provide an example in the Bayesian setting where VCG generates positive revenue and GSP has a Bayes-Nash equilibrium that generates zero revenue. Consider three players with iid valuations drawn uniformly from $[1,2]$ and three slots with $\alpha=[1,0.5,0.5]$. Let $v^{(i)}$ be the $i^{\text {th }}$ largest valuation (which is naturally a random variable defined by $\mathbf{v})$. We have

$$
\mathbb{E}\left[\mathcal{R}^{V C G}(\mathbf{v})\right]=\mathbb{E}\left[0.5 v^{(2)}\right]=\frac{3}{4} .
$$

Now, consider the following equilibrium of GSP: $b_{i}\left(v_{i}\right)=0$ for $i=2,3$ and $b_{1}\left(v_{1}\right)=v_{1}$. Clearly player 1 is in equilibrium. To see that players $i=2,3$ are in equilibrium, suppose player $i$ has valuation $v_{i}>0$. Then his expected utility when bidding any value in $[0,1]$ is $0.5 v_{i}$, whereas if he changed his bid to some $b>1$ his utility would be

$$
\begin{aligned}
\mathbb{E}\left[u_{i}\left(b^{\prime}, b_{-i}\right) \mid v_{i}\right] & =0.5 v_{i}+0.5 v_{i} \mathbb{P}\left(v_{1} \leq b^{\prime}\right)-\int_{0}^{b^{\prime}} v_{1} d \mathbb{P}\left(v_{1}\right)= \\
& =0.5 v_{i}+0.5 v_{i}\left(b^{\prime}-1\right)-\frac{\left(b^{\prime}\right)^{2}-1}{2} \leq \\
& \leq 0.5 v_{i} .
\end{aligned}
$$

Thus agent $i$ cannot increase his expected utility by placing a non-zero bid. 


\subsection{GSP with Myerson Reserve Price}

We now show that if valuations are drawn from a MHR distribution and GSP is paired with the Myerson reserve price, the resulting mechanism extracts a constant fraction of the optimal revenue.

Theorem 6 If valuations $v_{i}$ are drawn iid from a MHR distribution $F$ and $r$ is the Myerson reserve price for $F$, then $G S P$ with reserve price $r$ is guaranteed to extract a constant fraction $c$ of the optimal revenue.

We will obtain a constant $c \approx 6.06$. The main ingredient for proving this theorem is Myerson's Lemma, used together with the fact that, for MHR distributions, $\phi(x) \geq x-r$ for any $x \geq r$. To see this, note that

$$
x-\phi(x)=\frac{1-F(x)}{f(x)} \leq \frac{1-F(r)}{f(r)}=r
$$

by the definition of Myerson's reserve price.

Proof. Let $\mathcal{R}_{r}$ be the revenue of $\mathrm{GSP}_{r}$, and let $\nu(i)$ be the slot obtained by player $i$ in the optimal mechanism. Then we can write

$$
\begin{aligned}
\mathbb{E}\left[\mathcal{R}_{r}\right] & =\mathbb{E}\left[\sum_{i} \alpha_{\sigma(i)} \phi\left(v_{i}\right) \mathbb{1}\left\{v_{i} \geq r\right\}\right] \geq \\
& \geq \mathbb{E}\left[\sum_{i} \alpha_{\sigma(i)} v_{i} \mathbb{1}\left\{v_{i} \geq r\right\}\right]-\mathbb{E}\left[\sum_{i} \alpha_{\sigma(i)} r \mathbb{1}\left\{v_{i} \geq r\right\}\right] .
\end{aligned}
$$

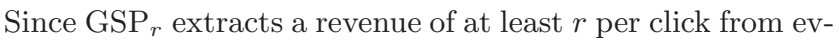
ery bidder with $v_{i}>r$, we have $\mathbb{E}\left[\mathcal{R}_{r}\right] \geq \mathbb{E}\left[\sum_{i} \alpha_{\sigma(i)} r \mathbb{1}\left\{v_{i} \geq\right.\right.$ $r\}]$. Adding these two inequalities, we get that $2 \mathbb{E}\left[\mathcal{R}_{r}\right]$ is at least the social welfare.

Suppose that, for distribution $F$, the social welfare generated by $\operatorname{GSP}_{r}$ is at least a $\beta$ fraction of the optimal social welfare. In this case, we know that

$$
\mathbb{E}\left[\sum_{i} \alpha_{\sigma(i)} v_{i} \mathbb{1}\left\{v_{i} \geq r\right\}\right] \geq \beta \mathbb{E}\left[\sum_{i} \alpha_{\nu(i)} v_{i} \mathbb{1}\left\{v_{i} \geq r\right\}\right]
$$

which is $\beta$-fraction of the social welfare generated by the Myerson mechanism. Summing all those statements, we conclude that the revenue of $\mathrm{GSP}_{r}$ is at least a $2 \beta$ fraction of Myerson's social welfare (and therefore also of Myerson's revenue). From $[5,16]$ we know that $\beta \leq 3.037$ (it is not hard to modify their proof to allow reserve prices). This gives us a factor of approximately 6.06 of the optimal revenue.

Notice that the proof above implies that when setting the Myerson reserve price, at least half of the social welfare is composed of revenue for the auctioneer.

\subsection{Well-separated click-through-rates}

Another way to bound the revenue of GSP without imposing reserve prices is to assume that the slot click-through-rates are well separated, in the sense of [13]. We say that clickthrough-rates are $\delta$-well separated if $\alpha_{i+1} \leq \delta \alpha_{i}$ for all $i$.

Lemma 7 If click-through-rates are $\delta$-well separated, then bidding $b_{i}\left(v_{i}\right)<(1-\delta) v_{i}$ is dominated by bidding $(1-\delta) v_{i}$.
Proof. Suppose player $i$ bids $b_{i}<(1-\delta) v_{i}$. If he increases his bid to $b_{i}^{\prime}=(1-\delta) v_{i}$ then with some probability he still gets the same slot (event $S$ ) and with some probability he gets a better slot (event $B$ ). Then clearly $\mathbb{E}\left[u_{i}\left(b_{i}, b_{-i}\right) \mid v_{i}\right] \leq \mathbb{E}\left[u_{i}\left(b_{i}^{\prime}, b_{-i}\right) \mid v_{i}\right]$ since the expectation conditioned to $S$ is the same and conditioned to $B$ it can only increase by changing the bid to $b_{i}^{\prime}$. To see that, let $\alpha_{\pi(i)}$ be the slot player $i$ gets under $b_{i}$ and $\alpha_{\pi^{\prime}(i)}$ the slot he gets under $b_{i}^{\prime}$. Conditioned on $B$ we know that $\alpha_{\pi^{\prime}(i)} \geq \delta^{-1} \alpha_{\pi(i)}$, and this generates value for bidder $i$ of at least $\alpha_{\pi^{\prime}(i)}\left(v_{i}-b_{i}^{\prime}\right)$, while the value with bid $b_{i}$ was at most $\alpha_{\pi(i)} v_{i}$, which implies the claim:

$$
\begin{aligned}
E\left[u_{i}\left(b_{i}, b_{-i}\right) \mid v_{i}, B\right] & \leq \mathbb{E}\left[\alpha_{\pi(i)} v_{i} \mid v_{i}, B\right] \leq \mathbb{E}\left[\delta \alpha_{\pi^{\prime}(i)} v_{i} \mid v_{i}, B\right]= \\
& =\mathbb{E}\left[\alpha_{\pi^{\prime}(i)}\left(v_{i}-(1-\delta) v_{i}\right) \mid v_{i}, B\right] \leq \\
& \leq \mathbb{E}\left[u_{i}\left(b_{i}^{\prime}, b_{-i}\right) \mid v_{i}, B\right] .
\end{aligned}
$$

Recall that under truthful bidding, the revenue of GSP is at least the revenue of VCG. If one eliminates the strategies $b_{i}\left(v_{i}\right)<(1-\delta) v_{i}$ from the players strategy set, then it is easy to see that any Bayesian-Nash equilibrium $\mathbf{b}$ has high revenue.

Corollary 8 If click-through-rates are $\delta$-well separated, and all players play undominated strategies, then

$$
\mathbb{E}_{\mathbf{v}}[\mathcal{R}(\mathbf{b}(\mathbf{v}))] \geq(1-\delta) \mathbb{E}_{\mathbf{v}}\left[\mathcal{R}^{V C G}(\mathbf{v})\right]
$$

Further, for any reserve price $r$, we also get

$$
\mathbb{E}_{\mathbf{v}}\left[\mathcal{R}_{r}(\mathbf{b})\right] \geq(1-\delta) \mathbb{E}_{\mathbf{v}}\left[\mathcal{R}_{r}^{V C G}(\mathbf{v})\right]
$$

Next we consider whether this bound on GSP revenue, with respect to the expected GSP revenue when all players report truthfully, continues to hold if agents do not eliminate dominated strategies. That is, we consider settings of limited rationality in which players may not be able to find dominated strategies. If we allow players to use dominated strategies, then we might have equilibria with very bad revenue compared to the expected revenue when agents bid truthfully, as one can see in the following example:

Example. Consider two players with iid valuations $v_{i} \sim$ Uniform $([0,1])$ and two slots with $\alpha=[1,1-\epsilon]$. Then VCG generates revenue $\mathbb{E}\left[\mathcal{R}^{V C G}(\mathbf{v})\right]=\mathbb{E}\left[\epsilon \min \left\{v_{1}, v_{2}\right\}\right]=O(\epsilon)$, and if agents report truthfully the GSP auction generates revenue $\mathbb{E}\left[\min \left\{v_{1}, v_{2}\right\}\right]=O(1)$. However, consider the following equilibrium:

$$
\begin{aligned}
& b_{1}\left(v_{1}\right)=\left\{\begin{array}{lr}
\epsilon(1-\delta), & v_{1} \geq \epsilon(1-\delta) \\
\epsilon v_{1}, & v_{1}<\epsilon(1-\delta)
\end{array}\right. \\
& b_{2}\left(v_{2}\right)=\left\{\begin{array}{lr}
\epsilon, & v_{2} \geq 1-\delta \\
\epsilon^{2}(1-\delta), & \epsilon(1-\delta) \leq v_{2}<1-\delta \\
\epsilon v_{2}, & v_{2}<\epsilon(1-\delta)
\end{array}\right.
\end{aligned}
$$

It is not hard to check that this is an equilibrium. In fact, for two player GSP in the Bayesian setting, playing $\left(\alpha_{1}-\right.$ $\left.\alpha_{2}\right) v_{i} / \alpha_{1}$ is a best reply - and any bid that gives the player the same outcome is also a best reply. So, in the above example, one can simply check that the bids generate the 
same utility as bidding $b_{i}\left(v_{i}\right)=\epsilon v_{i}$. This example generates revenue $\mathbb{E} \mathcal{R}(\mathbf{b})=O(\epsilon(\epsilon+\delta))$, so taking $\delta=O(\epsilon)$ in the above example give us $O\left(\epsilon^{2}\right)$ revenue.

However, this is a feature of having only 2 players, as shown in the following theorem, which is a version of Corollary 8 that doesn't depend on eliminating dominated strategies.

Theorem 9 With $n$ players with iid valuations $v_{i}$ and $\delta$ well separated click-through-rates, then for all Bayes-Nash equilibria $\mathbf{b}$ in which agents do not overbid,

$$
\mathbb{E}[\mathcal{R}(\mathbf{b})] \geq \frac{n-2}{n}(1-\delta) \mathbb{E}\left[\mathcal{R}^{V C G}(\mathbf{v})\right]
$$

Proof. We will prove the stronger result that the expected GSP revenue at equilibrium is within a factor of $\frac{n-2}{n}(1-\delta)$ of the expected GSP revenue when agents report truthfully. We first claim that, for a profile $\mathbf{b}$ in BayesianNash equilibrium and any two players $i$ and $j$, we have that

$$
\mathbb{P}_{v \sim F}\left[b_{i}(v)<(1-\delta) v-\epsilon, b_{j}(v)<(1-\delta) v-\epsilon\right]=0 .
$$

To see this, suppose the contrary. Then there is $\epsilon^{\prime} \ll \epsilon$ such that if we take $F^{\prime}=\left.F\right|_{\left[v^{0}-\epsilon^{\prime}, v^{0}+\epsilon^{\prime}\right]}$ then

$$
\mathbb{P}_{v \sim F^{\prime}}\left[b_{i}(v)<(1-\delta) v-\epsilon, b_{j}(v)<(1-\delta) v-\epsilon\right]>0 .
$$

For $\epsilon^{\prime}$ small enough $\underline{v_{0}}=v^{0}-\epsilon$ and some $\epsilon^{\prime \prime}<\epsilon$, we have

$$
\mathbb{P}_{v \sim F^{\prime}}\left[b_{i}(v)<(1-\delta) \underline{v_{0}}-\epsilon^{\prime \prime}, b_{j}(v)<(1-\delta) \underline{v_{0}}-\epsilon^{\prime \prime}\right]>0 .
$$

Now pick $v^{i}, v^{j}$ in this interval such that $\mathbb{P}_{v \sim F^{\prime}}\left[b_{i}\left(v^{i}\right) \leq\right.$ $b_{i}(v)<(1-\delta) \underline{\left.v_{0}\right]}>0$ and the same for $j$. By lemma $\overline{7}$, playing $(1-\delta) \overline{v^{i}}$ is a best response, then for player $j$ for example, it can't be the case that any of the other players play between $b_{j}\left(v^{j}\right)$ and $(1-\delta) v^{j}$ with positive probability. Therefore

$$
\begin{aligned}
& \mathbb{P}_{v \sim F^{\prime}}\left[b_{j}(v) \in\left[b_{i}\left(v^{i}\right),(1-\alpha) v^{i}\right)\right]=0 \\
& \mathbb{P}_{v \sim F^{\prime}}\left[b_{i}(v) \in\left[b_{j}\left(v^{j}\right),(1-\alpha) v^{j}\right)\right]=0
\end{aligned}
$$

but notice this is a contradiction. This completes the proof of the claim.

Now, we can think of the procedure of sampling $\mathbf{v}$ iid from $F$ in the following way: sample $v_{i}^{\prime \prime} \sim F$ iid, let $v_{i}^{\prime}$ be the sorted valuations, and then apply a random permutation $\tau \in S_{n}$ to the values so that $v_{i}=v_{\tau(i)}^{\prime}$. Notice that $\mathbf{v}$ is iid and now, notice that with $\geq 1-\frac{2}{n}$ probability, $v_{i}^{\prime}$ and $v_{i+1}^{\prime}$ will generate $(1-\delta) v_{i}^{\prime}$ and $(1-\delta) v_{i+1}^{\prime}$ bids producing $(1-\delta) \alpha_{i} v_{i+1}^{\prime}$ revenue, therefore

$$
\begin{aligned}
\mathbb{E}[\mathcal{R}(\mathbf{v})] & \geq \mathbb{E}\left[\sum_{i}\left(1-\frac{2}{n}\right)(1-\delta) \alpha_{i} v_{i+1}^{\prime}\right] \\
& \geq \frac{n-2}{n}(1-\delta) \mathbb{E}\left[\mathcal{R}^{V}(\mathbf{v})\right] .
\end{aligned}
$$

\section{REVENUE IN THE GSP HIERARCHY}

Up to this point we have been mainly concerned with comparing the VCG revenue (which is matched at one particular equilibrium of GSP) with the worst-case revenue over all possible equilibria of GSP. We have shown that there are equilibria of GSP that generate arbitrarily more and arbitrarily less revenue than VCG. We now come back to the full information setting to compare the revenue extraction properties of the different classes of GSP equilibria. Can one equilibrium class generate more or less revenue than another?

This question of comparing the revenue of VCG and envyfree equilibria of GSP was addressed by [7], who show that the revenue in any envy-free equilibrium is at least that of the VCG outcome (i.e. the VCG outcome is the envy-free equilibria generating smallest possible revenue). Moreover, as we've shown, an envy-free equilibrium can generate arbitrarily more revenue than the VCG outcome. Varian [21] shows how to compute the revenue optimal envy free Nash equilibrium, if we assume that agents will overbid. Allowing overbidding can result in very high revenue (eg.., the maximum valuation in a single item auction). Here we determine the maximum revenue that can be obtained if we do not assume that agents bid at envy-free equilibria, and without requiring that agents apply the dominated strategy of overbidding.

\subsection{Envy-free and efficient equilibrium}

As shown in the example of Figure 1, there are efficient equilibria that generate arbitrarily less revenue then any envyfree equilibrium. For the other direction, we show that all revenue-optimal equilibria are envy-free.

Theorem 10 For any AdAuctions instance such that $\alpha_{i}>$ $\alpha_{i+1} \forall i$, all revenue-optimal efficient equilibria are envy-free. Moreover, we can write the revenue optimal efficient equilibrium explicitly as function of $\alpha, \mathbf{v}$.

Proof. Given an efficient equilibrium $\mathbf{b}$, if it is not envyfree, we show that we can improve revenue by slightly increasing one of the bids. If the equilibrium is not envy-free, there is at least one player that envies the player above, i.e.

$$
\alpha_{i}\left(v_{i}-b_{i+1}\right)<\alpha_{i-1}\left(v_{i}-b_{i}\right) .
$$

As pointed out in [7], if in an efficient equilibrium no player envies the above slot (i.e. no player $i$ wants to take the above slot $i-1$ by the price per click player $i$ is paying) then the equilibrium is envy-free.

Let $i$ be the player with the smallest index that envies slot $i-1$. Consider the bid profile $\mathbf{b}^{\prime}$ such that $b_{j}^{\prime}=b_{j}$ for $j \neq i$ and $b_{i}^{\prime}=b_{i}+\epsilon$. We will verify that the Nash inequalities for player $i-1$ still hold when $\epsilon>0$ is sufficiently small. In other words, we will show that no Nash inequality for player $i-1$ holds with equality in $\mathbf{b}$.

For slots $j>i-1$, notice that

$$
\alpha_{j}\left(v_{i}-b_{j+1}\right) \leq \alpha_{i}\left(v_{i}-b_{i+1}\right)<\alpha_{i-1}\left(v_{i}-b_{i}\right)
$$

where the first is a standard Nash inequality and the second is the hypothesis that player $i$ envies the above slot. Now, since $v_{i-1}>v_{i}$ in an efficient equilibrium, we have

$$
\alpha_{j}\left(v_{i-1}-b_{j+1}\right)<\alpha_{i-1}\left(v_{i-1}-b_{i}\right) .
$$

For slots $j<i-1$, we use the fact that player $i$ is the first envious player. Also, without loss of generality, we can 
assume player 1 bids $v_{1}$. Therefore we need to verify the Nash inequalities only for $j=2,3, \ldots, k-1$. We have

$$
\alpha_{i-1}\left(v_{i}-b_{i}\right) \geq \alpha_{j}\left(v_{i}-b_{j+1}\right)>\alpha_{j}\left(v_{i}-b_{j}\right)
$$

where the first inequality comes from the fact that player $i-1$ doesn't envy any player $j$ above him and the second inequality comes from the fact that $b_{j}>b_{j+1}$, since otherwise the player in slot $j$ would envy the player in slot $j-1$.

In fact, we can give a more explicit proof of Theorem 10 by showing the bid profile that generates largest revenue and verifying it is an envy-free equilibrium. Given $(\alpha, \mathbf{v})$, we will define a bid profile $\mathbf{b}$ in a bottom up fashion:

$$
\begin{gathered}
b_{n}=\min \left\{v_{n}, \frac{\alpha_{n-1}-\alpha_{n}}{\alpha_{n-1}} v_{n-1}\right\}, \\
b_{i}=\min \left\{v_{i}, \frac{\alpha_{i-1}-\alpha_{i}}{\alpha_{i-1}} v_{i-1}+\frac{\alpha_{i}}{\alpha_{i-1}} b_{i+1}\right\} \forall i<n .
\end{gathered}
$$

We need to show the following things about this bid profile b: (i) it is in Nash equilibrium, (ii) it is envy free, and (iii) no other efficient Nash equilibrium generates higher revenue. Begin by noticing that if $\mathbf{b}$ is Nash, then player $i-1$ doesn't want to take slot $i$, for all $i$, and therefore $\alpha_{i-1}\left(v_{i-1}-b_{i}\right) \geq \alpha_{i}\left(v_{i-1}-b_{i+1}\right)$ and this is satisfied by definition by the bid vector presented. Notice also that this series of inequalities implies an upper bound on the maximum revenue in an efficient equilibrium and this bound is achieved exactly by the bid profile defined above.

Furthermore, for all $j \leq i-1$ we have $\alpha_{i-1}\left(v_{j}-b_{i}\right) \geq$ $\alpha_{i}\left(v_{j}-b_{i+1}\right)$ therefore by composing this expression with different values of $i$ and $j$, it is straightforward to show that no player can profit by decreasing his bid. We prove that no player can profit by overbidding as a simple corollary of envy-freeness. For that, we need to prove that

$$
\alpha_{i}\left(v_{i}-b_{i+1}\right) \geq \alpha_{i-1}\left(v_{i}-b_{i}\right) .
$$

If $b_{i}=v_{i}$ than this is trivial. If not, then substitute the expression for $b_{i}$ and notice it reduces to $v_{i-1} \geq v_{i}$. Now, this proved local envy-freeness, what implies that no player wants the slot above him by the price he player above him is paying. This in particular implies that no player wants to increase his bid to take a slot above.

\subsection{Cost of efficiency}

Here, we analyze the relation between revenue and efficiency in GSP auctions. One might ask if it is possible to have optimal efficiency and optimal revenue in the same equilibrium. In other words, among all GSP equilibria, is the revenue-maximizing equilibrium necessarily efficient? We give a negative answer to this question, showing that for some AdAuction instances, we can increase revenue by selecting an inefficient equilibrium. However, we give a natural sufficient condition so that the revenue-optimal equilibrium is efficient.

We define the cost of efficiency for a given click-through-rate as the ratio:

$$
\operatorname{CoE}(\alpha)=\max _{\mathbf{v}} \frac{\max _{\mathbf{b} \in \operatorname{NaSh}(\alpha, \mathbf{v})} \mathcal{R}(\mathbf{b})}{\max _{\mathbf{b} \in \operatorname{EFFNASH}(\alpha, \mathbf{v})} \mathcal{R}(\mathbf{b})}
$$

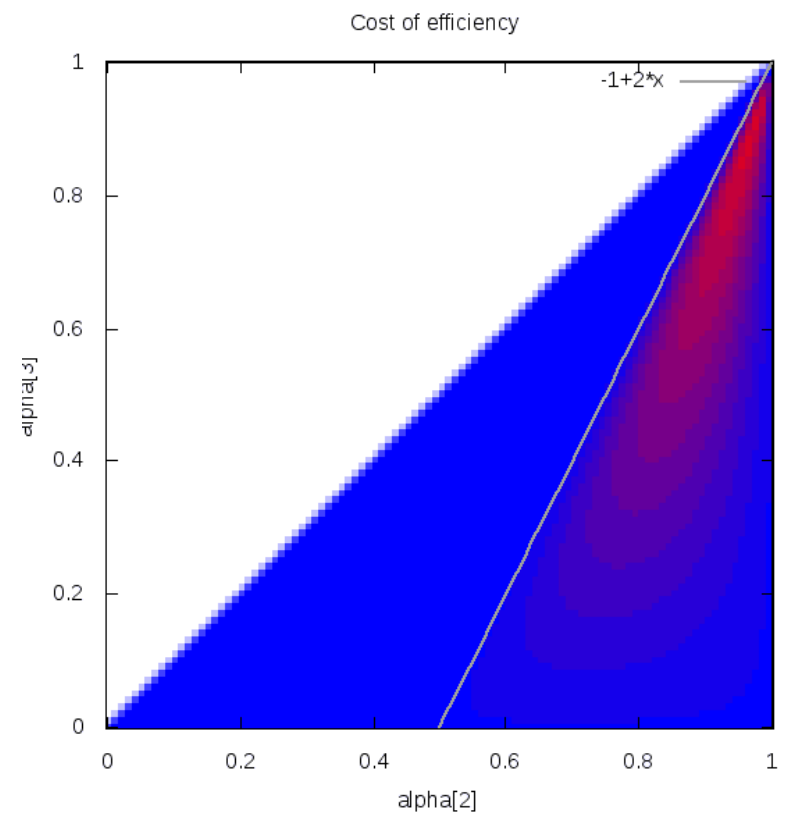

Figure 2: Cost of efficiency for $\alpha=\left[1, \alpha_{2}, \alpha_{3}\right]$ : in the plot, blue means 1.0 and red means 1.1 .

where NASH is the set of all bid profiles in Nash equilibrium and EFFNASH is the set of all efficient Nash equilibrium. In Figure 2 we calculate this value empirically for each $\alpha=$ $\left[1, \alpha_{2}, \alpha_{3}\right]$, where each $\alpha_{i}$ is an integer multiple of 0.01 in $[0,1]$. In all cases we found that $1 \leq \operatorname{CoE}(\alpha)<1.1$. The color of $\left(\alpha_{1}, \alpha_{2}\right)$ in the graph corresponds to $\operatorname{CoE}\left(1, \alpha_{2}, \alpha_{3}\right)$, where blue represents 1 and red represents 1.1. By solving a constrained non-linear optimization problem, one can show that the worst CoE for 3 slots is 1.09383 .

Example. One example where an inefficient equilibrium generates strictly more revenue then all efficient ones is $\alpha=\left[1, \frac{2}{3}, \frac{1}{6}\right]$ and $v=\left[1, \frac{7}{8}, \frac{6}{8}\right]$ : the best efficient revenue is given by $\frac{1}{3}+\frac{7}{8} \approx 1.20833$ (which can be calculated using the formula in the last section), but for the (inefficient) allocation $\pi=[2,1,3]$ there is an equilibrium generating revenue 1.21528 .

Note that Figure 2 seems to imply that $\operatorname{CoE}(\alpha)=1$ whenever $\alpha_{1}-\alpha_{2} \geq \alpha_{2}-\alpha_{3}$. This motivates us to look at AdAuctions instances with convex click-through-rates, i.e., $\alpha_{i}-\alpha_{i+1} \geq \alpha_{i+1}-\alpha_{i+2}$. Notice that this is a natural assumption, since most models for CTR follow convexity, such as exponential CTR as in [13] and Markovian user models as in [1]. We prove that convexity of click-through rates does indeed imply that $\mathrm{CoE}=1$.

Theorem 11 If click-through-rates $\alpha$ are convex (i.e. $\alpha_{i}-$ $\left.\alpha_{i+1} \geq \alpha_{i+1}-\alpha_{i+2}, \forall i\right)$, then there is a revenue maximizing equilibrium that is also efficient.

Proof. Let $\mathbf{b}$ be the revenue maximizing efficient Nash equilibrium, which can be calculated according to the formula in the last section. Now, fix an allocation $\pi$ and let 
$\mathbf{b}^{\prime}$ be an equilibrium under allocation $\pi$. We say that $\mathbf{b}$ is saturated for slot $i$ if $b_{i}=v_{i}$. First we prove the theorem under the simplifying assumption that no slot is saturated in the maximum revenue equilibrium. We then prove the general case, which is more technical.

Under the no-saturation assumption, we have

$$
\mathcal{R}(\mathbf{b})=\sum_{i} \alpha_{i} b_{i+1}=\sum_{i} \sum_{j \geq i}\left(\alpha_{j}-\alpha_{j+1}\right) v_{j}
$$

Notice that we can view this expression as a dot product of two vectors where one has elements of the form $v_{i}$ and other has elements in the form $\alpha_{j}-\alpha_{j+1}$. Notice also that due to the convexity assumption, this is a dot product of two sorted vectors. Now, for $\mathbf{b}^{\prime}$, we will bound revenue as follows. Define $m(\pi, i, j)=\max \{\pi(i), \pi(i+1), \pi(i+2), \ldots, \pi(j)\}$. Let $p$ be such that the $k=i, i+1, \ldots, i+p$ are all the indices such that $m(\pi, i, k)=\pi(i)$. Now, notice that the player in slot $i$ doesn't want to take slot $i+p+1$, so

$$
\alpha_{i}\left(v_{\pi(i)}-b_{\pi(i+1)}^{\prime}\right) \geq \alpha_{i+p+1}\left(v_{\pi(i)}-b_{\pi(i+p+2)}^{\prime}\right) .
$$

This implies

$$
\begin{aligned}
\alpha_{i} b_{\pi(i+1)}^{\prime} & \leq \alpha_{i+p+1} b_{\pi(i+p+2)}^{\prime}+\left(\alpha_{i}-\alpha_{i+p+1}\right) v_{\pi(i)}= \\
& =\alpha_{i+p+1} b_{\pi(i+p+2)}^{\prime}+\sum_{j=i}^{i+p}\left(\alpha_{j}-\alpha_{j+1}\right) v_{m(\pi, i, j)} .
\end{aligned}
$$

We can now apply recursion to conclude that

$$
\mathcal{R}\left(\mathbf{b}^{\prime}\right)=\sum_{i} \alpha_{i} b_{\pi(i+1)}^{\prime} \leq \sum_{i} \sum_{j \geq i}\left(\alpha_{j}-\alpha_{j+1}\right) v_{m(\pi, i, j)}
$$

Now, notice that equation (3) can also be written as a dot product between two vectors of type $v_{i}$ and $\alpha_{j}-\alpha_{j+1}$. If we sort the vectors, we see that the $\left(\alpha_{j}-\alpha_{j+1}\right)$-vector is the same and the sorted vector of $v_{j}$ for equation (3) is dominated by that of equation (2), in the sense that it is pointwise smaller. To see that, simply count how many times we have one of $v_{1}, \ldots, v_{i}$ appear in both vectors for each index $i$. For equation (2) they appear $\sum_{j=1}^{i} j$ times. For equation (3), they appear at most

$$
\sum_{j=1}^{i} 1+\max \{p ; m(\pi(j, j+p)) \leq i\} \leq \sum_{j=1}^{i} j
$$

times. Since the $\left(\alpha_{j}-\alpha_{j+1}\right)$-vectors are the same in both equations, the $v_{i}$ vector in the first equation dominates the order and in the first equation both vectors are sorted in the same order, so it must be the case that $\mathcal{R}(\mathbf{b}) \geq \mathcal{R}\left(\mathbf{b}^{\prime}\right)$.

Case with saturations: We now remove our simplifying assumption and prove the general result. Let $\mathbf{b}$ be the optimal efficient equilibrium and let $S \subseteq[n+1]$ be the set of saturated bids, including $n+1$ (where we consider a "fake" player $n+1$ with $b_{n+1}=v_{n+1}=0$ ), i.e., $i \in S$ iff $b_{i}=v_{i}$. Let $S(i)=\min \{j \in S ; j>i\}$.

Given an allocation $\pi$, we wish to define an upper bound, $\overline{\mathcal{R}}_{\pi}$, on the revenue of a bid profile that induces allocation $\pi$ at equilibrium. To this end, we define

$$
B_{\pi}(j)=\left\{\begin{array}{c}
\alpha_{S(j)-1} v_{S(j)}+\sum_{i=\sigma(j)}^{S(j)-2}\left(\alpha_{i}-\alpha_{i+1}\right) v_{m(\pi, \sigma(j), i)} \\
\text { if } \sigma(j) \leq S(j)-1 \\
\alpha_{S(j)-1} v_{S(j)}-v_{j}\left(\alpha_{S(j)-1}-\alpha_{\sigma(j)}\right) \\
\text { if } \sigma(j) \geq S(j)-1
\end{array}\right.
$$

We then define

$$
\overline{\mathcal{R}}_{\pi}=\sum_{j} B_{\pi}(j)
$$

We claim that this is, indeed, an upper bound on revenue. Moreover, this bound is tight for revenue at efficient equilibria (i.e. when $\pi$ is the identity $i d$ ).

Claim 12 If bid profile $\mathbf{b}$ induces allocation $\pi$ at equilibrium, then $\mathcal{R}(\mathbf{b}) \leq \overline{\mathcal{R}}_{\pi}$.

Claim 13 There exists an efficient equilibrium with revenue $\overline{\mathcal{R}}_{i d}$.

Using these two claims we want to argue that $i d$ is the permutation that maximizes $\overline{\mathcal{R}}_{\pi}$ and therefore we can show that for all inefficient bid profile $\mathbf{b}^{\prime}$ we have

$$
\mathcal{R}\left(\mathbf{b}^{\prime}\right) \leq \overline{\mathcal{R}}_{\pi} \leq \overline{\mathcal{R}}_{i d}=\mathcal{R}(\mathbf{b}) .
$$

To show this, consider some permutation $\pi$. Let $j=\max \{k ; \pi(k) \neq$ $k\}$ and define a permutation $\pi^{\prime}$ such that $\pi^{\prime}(k)=k$ for $k \geq j$ and $\pi^{\prime}(k)=\pi(k)$ for $k<\sigma(j)$ and $\pi^{\prime}(k)=\pi(k+1)$ for $\sigma(j) \leq k<j$. Essentially this is picking the last player that is not allocated to his correct slot and bring him there. Now, if we prove that $\overline{\mathcal{R}}_{\pi^{\prime}} \geq \overline{\mathcal{R}}_{\pi}$, then we are done, since we can repeat this procedure many times and get to $i d$.

Claim $14 \overline{\mathcal{R}}_{\pi^{\prime}} \geq \overline{\mathcal{R}}_{\pi}$

This completes the proof, subject to our claims.

Proof of Claim 12 : We will show that for all $\mathbf{b}^{\prime}$ inducing allocation $\pi$, we have $\alpha_{\sigma(j)} b_{\sigma(j)+1}^{\prime} \leq B_{\pi}(j)$. For $\sigma(j)=$ $S(j)-1$, we use the fact that $b_{\sigma(j)+1}^{\prime}=b_{S(j)}^{\prime} \leq v_{S(j)}$. For $\sigma(j)<S(j)-1$ the result follows in the same way as in the unsaturated case. For $\sigma(j)>S(j)-1$, we use the fact that player $j$ doesn't want to take slot $j$ and therefore

$\alpha_{\sigma(j)}\left(v_{j}-b_{\sigma(j)+1}^{\prime}\right) \geq \alpha_{S(j)-1}\left(v_{j}-b_{S(j)-1}^{\prime}\right) \geq \alpha_{S(j)-1}\left(v_{j}-v_{S(j)}\right)$ since

$$
b_{S(j)}^{\prime} \leq \min \left\{v_{\pi(1)}, \ldots, v_{\pi(S(j)-1)}\right\} \leq v_{S(j)}
$$

and $\sigma(j)>S(j)-1$ so one of the players with value $\leq v_{S(j)}$ must be among the first $S(j)-1$ slots. Reordering the Nash inequalities above gives us the desired result.

Proof of Claim 13 : This claim follows from the formula defining the optimal-revenue efficient equilibrium in the previous section.

Proof of Claim 14: Note first that $B_{\pi}(k)=B_{\pi^{\prime}}(k)$ for all $k>j$. Moreover, for any $k$ with $\sigma(k)<\sigma(j)$, we will 
have $\sigma^{\prime}(k)=\sigma(k)$. In this case, either $S(k)<\sigma(k)$ in which case $B_{\pi^{\prime}}(k)=B_{\pi}(k)$, or else

$$
\begin{aligned}
B_{\pi^{\prime}}(k) & =\alpha_{S(k)-1} v_{S(k)}+\sum_{i=\sigma(k)}^{S(k)-2}\left(\alpha_{i}-\alpha_{i+1}\right) v_{m(\pi, \sigma(k), i)} \\
& \geq \alpha_{S(k)-1} v_{S(k)}+\sum_{i=\sigma^{\prime}(k)}^{S(k)-2}\left(\alpha_{i}-\alpha_{i+1}\right) v_{m\left(\pi^{\prime}, \sigma^{\prime}(k), i\right)} \\
& =B_{\pi^{\prime}}(k) .
\end{aligned}
$$

It remains to consider $k$ is such that $\sigma(j) \leq \sigma(k) \leq j$; that is, those players $k$ such that $\sigma(k) \neq \sigma^{\prime}(k)$. For each such player, we will consider the difference between $B_{\pi}(k)$ and $B_{\pi^{\prime}}(k)$. First note that, for player $j$, we have

$$
\begin{aligned}
& B_{\pi}(j)-B_{\pi^{\prime}}(j) \\
& =\left(\alpha_{S(j)-1} v_{S(j)}+\sum_{i=\sigma(j)}^{S(j)-2}\left(\alpha_{i}-\alpha_{i+1}\right) v_{m(\pi, \sigma(j), i)}\right) \\
& -\left(\alpha_{S(j)-1} v_{S(j)}+\sum_{i=\sigma^{\prime}(j)}^{S(j)-2}\left(\alpha_{i}-\alpha_{i+1}\right) v_{m\left(\pi^{\prime}, \sigma^{\prime}(j), i\right)}\right) \\
& =\sum_{i=\sigma(j)}^{j-1}\left(\alpha_{i}-\alpha_{i+1}\right) v_{j}
\end{aligned}
$$

For $k \neq j$, we claim that $B_{\pi^{\prime}}(k)-B_{\pi}(k) \geq v_{j}\left(\alpha_{\sigma(k)-1}-\right.$ $\left.\alpha_{\sigma(k)}\right)$. We proceed by two cases. First, if $S(k) \leq \sigma(k)$, we have

$$
\begin{aligned}
B_{\pi^{\prime}}(k)-B_{\pi}(k)= & \left(\alpha_{S(k)-1} v_{S(k)}-v_{k}\left(\alpha_{S(k)-1}-\alpha_{\sigma^{\prime}(k)}\right)\right) \\
& -\left(\alpha_{S(k)-1} v_{S(k)}-v_{k}\left(\alpha_{S(k)-1}-\alpha_{\sigma(k)}\right)\right) \\
= & v_{k}\left(\alpha_{\sigma(k)-1}-\alpha_{\sigma(k)}\right) \\
\geq & v_{j}\left(\alpha_{\sigma(k)-1}-\alpha_{\sigma(k)}\right)
\end{aligned}
$$

Second, if $S(k)-1>\sigma(k)$, then we have

$$
\begin{aligned}
B_{\pi^{\prime}}(k)-B_{\pi}(k) & \left(\alpha_{S(k)-1} v_{S(k)}+\sum_{i=\sigma^{\prime}(k)}^{S(k)-2}\left(\alpha_{i}-\alpha_{i+1}\right) v_{m\left(\pi^{\prime}, \sigma^{\prime}(k), i\right)}\right) \\
& -\left(\alpha_{S(k)-1} v_{S(k)}+\sum_{i=\sigma(k)}^{S(k)-2}\left(\alpha_{i}-\alpha_{i+1}\right) v_{m(\pi, \sigma(k), i)}\right) \\
= & \left(\alpha_{S(k)-2}-\alpha_{S(k)-1}\right) v_{m\left(\pi^{\prime}, \sigma^{\prime}(k), S(k)-2\right)} \\
& +\sum_{i=\sigma^{\prime}(k)}^{S(k)-3} v_{m\left(\pi^{\prime}, \sigma^{\prime}(k), i\right)}\left[\left(\alpha_{i}-\alpha_{i+1}\right)-\left(\alpha_{i+1}-\alpha_{i+2}\right)\right] \\
\geq & v_{j}\left(\alpha_{S(k)-2}-\alpha_{S(k)-1}\right) \\
& +\sum_{i=\sigma^{\prime}(k)}^{S(k)-3} v_{j}\left[\left(\alpha_{i}-\alpha_{i+1}\right)-\left(\alpha_{i+1}-\alpha_{i+2}\right)\right] \\
= & v_{j}\left(\alpha_{\sigma(k)-1}-\alpha_{\sigma(k)}\right)
\end{aligned}
$$

Notice that we strongly use the fact that click-through-rates are convex in the last inequality to ensure that $\left(\alpha_{i}-\alpha_{i+1}\right)-$ $\left(\alpha_{i+1}-\alpha_{i+2}\right) \geq 0$.
Therefore, taking the sum over all $k$ with $\sigma(j) \leq \sigma(k) \leq j$, we have

$$
\begin{aligned}
\sum_{k: \sigma(j)<\sigma(k) \leq j}\left(B_{\pi^{\prime}}(k)-B_{\pi}(k)\right) & \geq \sum_{i=\sigma(j)}^{j-1} v_{j}\left(\alpha_{i}-\alpha_{i+1}\right) \\
& =B_{\pi}(j)-B_{\pi^{\prime}}(j)
\end{aligned}
$$

so that

$$
\sum_{k: \sigma(j) \leq \sigma(k) \leq j}\left(B_{\pi^{\prime}}(k)-B_{\pi}(k)\right) \geq 0 .
$$

Combining this with the fact that $B_{\pi^{\prime}}(k) \geq B_{\pi}(k)$ for all $k$ with $\sigma(k)<\sigma(j)$ or $\sigma(k)>j$, we conclude

$$
\overline{\mathcal{R}}_{\pi^{\prime}}=\sum_{k} B_{\pi^{\prime}}(k) \geq \sum_{k} B_{\pi}(k)=\overline{\mathcal{R}}_{\pi}
$$

as desired.

\section{REFERENCES}

[1] G. Aggarwal, J. Feldman, S. Muthukrishnan, and M. Pál. Sponsored search auctions with markovian users. In Proceedings of the 4th International Workshop on Internet and Network Economics, WINE '08, pages 621-628, Berlin, Heidelberg, 2008. Springer-Verlag.

[2] G. Aggarwal, A. Goel, and R. Motwani. Truthful auctions for pricing search keywords. In $E C$ '06: Proceedings of the rth ACM conference on Electronic commerce, pages 1-7, New York, NY, USA, 2006. ACM.

[3] S. Athey and D. Nekipelov. Equilibrium and uncertainty in sponsored search advertising. In AdAuctions Workshop 2010, 2010.

[4] T. Borgers, I. Cox, M. Pesendorfer, and V. Petricek. Equilibrium bids in sponsored search auctions: Theory and evidence. Technical report, 2007.

[5] I. Caragiannis, P. Kanellopoulos, C. Kaklamanis, and M. Kyropoulou. On the efficiency of equilibria in generalized second price auctions. In EC'11, 2011.

[6] B. Edelman and M. Ostrovsky. Strategic bidder behavior in sponsored search auctions. Decision Support Systems, (43):192-198, 2007.

[7] B. Edelman, M. Ostrovsky, and M. Schwarz. Internet advertising and the generalized second-price auction: Selling billions of dollars worth of keywords. The American Economic Review, 97(1):242-259, March 2007.

[8] B. Edelman and M. Schwarz. Optimal auction design in a multi-unit environment: The case of sponsored search auctions. Technical report, 2006.

[9] B. Edelman and M. Schwarz. Optimal auction design and equilibrium selection in sponsored search auctions. American Economic Review, (2), 2010.

[10] J. Feng, H. K. Bhargava, and D. M. Pennock. Implementing sponsored search in web search engines: Computational evaluation of alternative mechanisms. INFORMS J. on Computing, 19(1):137-148, 2007.

[11] R. D. Gomes and K. S. Sweeney. Bayes-nash equilibria of the generalized second price auction. In $E C$ ' 09 : Proceedings of the tenth ACM conference on Electronic commerce, pages 107-108, New York, NY, USA, 2009. ACM. 
[12] J. Hegeman. Facebook's ad auction. Talk at Ad Auctions Workshop, May 2010, 2010.

[13] S. Lahaie. An analysis of alternative slot auction designs for sponsored search. In EC'06: Proceedings of the 7th ACM conference on Electronic commerce, pages 218-227, New York, NY, USA, 2006. ACM.

[14] S. Lahaie, D. Pennock, A. Saberi, and R. Vohra. Algorithmic Game Theory, chapter Sponsored search auctions, pages 699-716. Cambridge University Press, 2007.

[15] S. Lahaie and D. M. Pennock. Revenue analysis of a family of ranking rules for keyword auctions. In Proceedings of the 8th ACM conference on Electronic commerce, EC '07, pages 50-56, New York, NY, USA, 2007. ACM.

[16] B. Lucier and R. Paes Leme. Gsp auctions with correlated types. In $E C^{\prime} 11,2011$.

[17] R. Myerson. Optimal auction design. Mathematics of Operations Research, 6(1):58-73, 1981.

[18] R. Paes Leme and E. Tardos. Pure and bayes-nash price of anarchy for generalized second price auctions. In FOCS10: 51st Annual IEEE Symposium on Foundations of Computer Science, 2010.

[19] H. Varian. Mechanism design double feature. Talk at New York Computer Science and Economics Day, October 2010, 2010.

[20] H. R. Varian. Position auctions. International Journal of Industrial Organization, 2006.

[21] H. R. Varian. Online ad auctions. American Economic Review Papers and Proceedings, 2009. 